\title{
Serine phosphorylation of cortactin is required for maximal host cell invasion by Campylobacter jejuni
}

\author{
Derrick R Samuelson and Michael E Konkel*
}

\begin{abstract}
Background: Campylobacter jejuni causes acute disease characterized by severe diarrhea containing blood and leukocytes, fever, and abdominal cramping. Disease caused by C. jejuni is dependent on numerous bacterial and host factors. C. jejuni invasion of the intestinal epithelial cells is seen in both clinical samples and animal models indicating that host cell invasion is, in part, necessary for disease. C. jejuni utilizes a flagellar Type III Secretion System (T3SS) to deliver the Campylobacter invasion antigens (Cia) to host cells. The Cia proteins modulate host cell signaling leading to actin cytoskeleton rearrangement necessary for C. jejuni host cell invasion, and are required for the development of disease.

Results: This study was based on the hypothesis that the $C$. jejuni CiaD effector protein mediates Erk 1/2 dependent cytoskeleton rearrangement. We showed that CiaD was required for the maximal phosphorylation of Erk $1 / 2$ by performing an immunoblot with a p-Erk 1/2 specific antibody and that Erk 1/2 participates in C. jejuni invasion of host cells by performing the gentamicin protection assay in the presence and absence of the PD98059 (a potent inhibitor of Erk $1 / 2$ activation). CiaD was also found to be required for the maximal phosphorylation of cortactin S405 and S418, as judged by immunoblot analysis. The response of human INT 407 epithelial cells to infection with C. jejuni was evaluated by confocal microscopy and scanning electron microscopy to determine the extent of membrane ruffling. This analysis revealed that $\mathrm{CiaD}$, Erk 1/2, and cortactin participate in C. jejuni-induced membrane ruffling. Finally, cortactin and N-WASP were found to be involved in C. jejuni invasion of host cells using siRNA to N-WASP, and siRNA to cortactin, coupled with the gentamicin protection assay.

Conclusion: We conclude that $\mathrm{CiaD}$ is involved in the activation of Erk $1 / 2$ and that activated Erk $1 / 2$ facilitates C. jejuni invasion by phosphorylation of cortactin on serine 405 and 418 . This is the first time that cortactin and N-WASP have been shown to be involved in C. jejuni invasion of host cells. These data also provide a mechanistic basis for the requirement of Erk 1/2 in C. jejuni-mediated cytoskeletal rearrangement.
\end{abstract}

Keywords: Bacterial pathogenesis, Effector protein, Invasion, Erk 1/2, N-WASP

\section{Lay abstract}

Campylobacter jejuni uses the flagellum as a Type III Secretion System (T3SS). A subset of proteins are exported from the flagellum and delivered to the cytosol of host cells where they modify host cell signaling events to promote bacterial invasion. Here we report that C. jejuni, which is the leading bacterial cause of food-borne disease worldwide, usurps the host cell signaling proteins Erk $1 / 2$ and cortactin. We show that the $C$. jejuni $\mathrm{CiaD}$

\footnotetext{
* Correspondence: konkel@vetmed.wsu.edu

School of Molecular Biosciences, Washington State University, College of Veterinary Medicine, Life Sciences Bldg. Room 302c, Pullman, Washington
} 99164-7520, USA

(c) 2013 Samuelson and Konkel; licensee BioMed Central Ltd. This is an open access article distributed under the terms of the Creative Commons Attribution License (http://creativecommons.org/licenses/by/2.0), which permits unrestricted use, distribution, and reproduction in any medium, provided the original work is properly cited. protein is required for the invasion of host cells and for the activation of Erk 1/2 (a host cell kinase) and cortactin (a cellular scaffolding protein). The characterization of a virulence protein and the identification of a novel host cell signaling pathway exploited by $C$. jejuni provides a significant advancement in the understanding of C. jejuni pathogenesis.

\section{Background}

Cortactin is an actin-binding protein that plays an integral role in the regulation and dynamics of the actin cytoskeleton. Cortactin has emerged as a key cellular protein that microbes readily subvert during the establishment of infection [1]. To date, cortactin has been demonstrated to
C Biomed Central 
be essential for the development of disease by numerous bacterial pathogens. While several pathogens, including Shigella, Neisseria, Rickettsia, Chlamydia, Staphylococcus, Listeria, Helicobacter, Escherichia, and Coxiella, require Src-mediated tyrosine phosphorylation of cortactin for host cell invasion, the mechanism of cortactin activation has only been partially elucidated or is, in most instances, not known [1-8]. The role of various actin cytoskeleton regulators, including Erk 1/2 and cortactin, in C. jejuni invasion has not been elucidated.

Campylobacter jejuni is a Gram-negative bacterial pathogen that causes acute disease characterized by severe diarrhea. $C$. jejuni causes $\sim 1.4$ to 2.3 million cases of gastroenteritis in the United States each year [9]. GuillainBarré syndrome (GBS), an autoimmune disease affecting the peripheral nervous system, can be a possible sequelae associated with certain strains of $C$. jejuni [10]. Motility, adherence, invasion, intracellular survival, and toxin production have all been shown to contribute to the severity of $C$. jejuni-mediated disease, illustrating the fact that disease is a multifactorial process [11-16].

Maximal cell invasion requires the Campylobacter invasion antigens (Cia) [14]. The Cia proteins are exported from the bacterium's flagellar Type III Secretion System (T3SS) and are delivered to the target host cell, where they presumably modify host cell regulatory proteins to promote $C$. jejuni-host cell entry and intracellular survival. Co-culture of $C$. jejuni with either host cells or host-like conditions results in increased expression of the genes encoding the Cia proteins $[17,18]$. To date, four Cia proteins have been identified, designated $\mathrm{CiaB}, \mathrm{CiaC}, \mathrm{CiaD}$, and CiaI $[11,16,19,20]$. The importance of the Cia proteins in campylobacteriosis has been demonstrated using in vivo studies with a $c i a B$ mutant, which is deficient in the secretion of all of the Cia proteins [15]. Piglets inoculated with a $C$. jejuni wild-type strain develop severe diarrhea within 24 hours of infection and exhibited major histological abnormalities, such as villus blunting and production of exudates in the lumen. In contrast, piglets inoculated with the ciaB mutant did not developed diarrhea until 3 days post infection and only exhibited minor histological lesions. Piglets inoculated with a $c i a B$ mutant harboring a wildtype copy of the $c i a B$ gene exhibited clinical signs of disease similar to piglets inoculated with the $C$. jejuni wild-type strain [21]. Given that the Cia proteins contribute to the development of $C$. jejuni-mediated enteritis, additional work is warranted to further dissect the functions of these proteins.

We recently identified a secreted protein, which we termed $\mathrm{CiaD}$ (Cj0788), that is exported from the flagellum and delivered to the cytosol of host cells. We found that $\mathrm{CiaD}$ activates the host cell kinases p38 and Erk 1/2, resulting in the secretion of interleukin-8 (IL-8) from host cells. Similarly, we found that CiaD-mediated activation of p38 and Erk 1/2 are required for maximal invasion of host cells by $C$. jejuni. CiaD function within host cells is dependent on a mitogen-activated protein (MAP) kinasedocking motif. Finally, $\mathrm{CiaD}$ was found to contribute to the development of disease, as evidenced by gross pathology and histopathology of tissues from IL-10 knockout mice inoculated with a $C$. jejuni wild-type strain, ciaD mutant, and ciaD complemented isolate [22]. While it is known that $\mathrm{CiaD}$ contributes to $C$. jejuni invasion of host cells, the details of the molecular mechanisms of $C$. jejuni-host cell invasion are incomplete.

The goal of this study was to determine the role of $\mathrm{CiaD}$ in C. jejuni host cell invasion. We hypothesized that the C. jejuni effector protein $\mathrm{CiaD}$ contributes to bacterial invasion by stimulation of Erk $1 / 2$ and the phosphorylation of cortactin. We sought to identify the role of Erk 1/2 and cortactin in C. jejuni invasion of host cells. More specifically, we sought to determine if the phosphorylation of cortactin is necessary for C. jejuni invasion of host cells, and whether $\mathrm{CiaD}$ contributes to the phosphorylation of cortactin.

\section{Results}

Erk $1 / 2$ and the $C$. jejuni CiaD effector protein are required for maximal invasion of human INT 407 epithelial cells

Experiments were initially performed to determine if $\mathrm{CiaD}$ contributes to the activation of the Erk $1 / 2$ signaling pathway. Consistent with previous work [22], we found that $\mathrm{CiaD}$ is required for maximal invasion of host INT 407 cells (Figure 1A) and contributes to the complete activation of the host cell kinase Erk 1/2 (Figure 1B). INT 407 cells are a human epithelial cell line [23]. To determine if Erk $1 / 2$ is involved in bacterial invasion, we performed a gentamicin protection assay in the presence of the MEK 1/2 inhibitor PD98059 (a potent inhibitor of Erk $1 / 2$ activation). Inhibition of Erk $1 / 2$ activation was found to significantly decrease the number of $C$. jejuni internalized (Figure 1C), which is consistent with previous reports $[24,25]$. These results demonstrate that $\mathrm{CiaD}$ and Erk $1 / 2$ are necessary for maximal host cell invasion by C. jejuni.

\section{CiaD is required for host cell membrane ruffling independent of Rho GTPase activation}

Three Rho GTPases (i.e., Rho, Rac1, and Cdc42) are involved in the regulation and dynamic rearrangement of the actin cytoskeleton [26]. C. jejuni invasion of host cells is accompanied by the activation of the Rho GTPases Rac1 and Cdc42 [27,28]. Rac1 is involved in the formation of lamellipodia and Cdc42 is involved in the formation of filopodia [26]. We will refer to lamellipodia and filopodia extensions, which are membrane protrusions associated with reorganization of actin microfilaments, as membrane ruffles throughout the manuscript. Experiments 


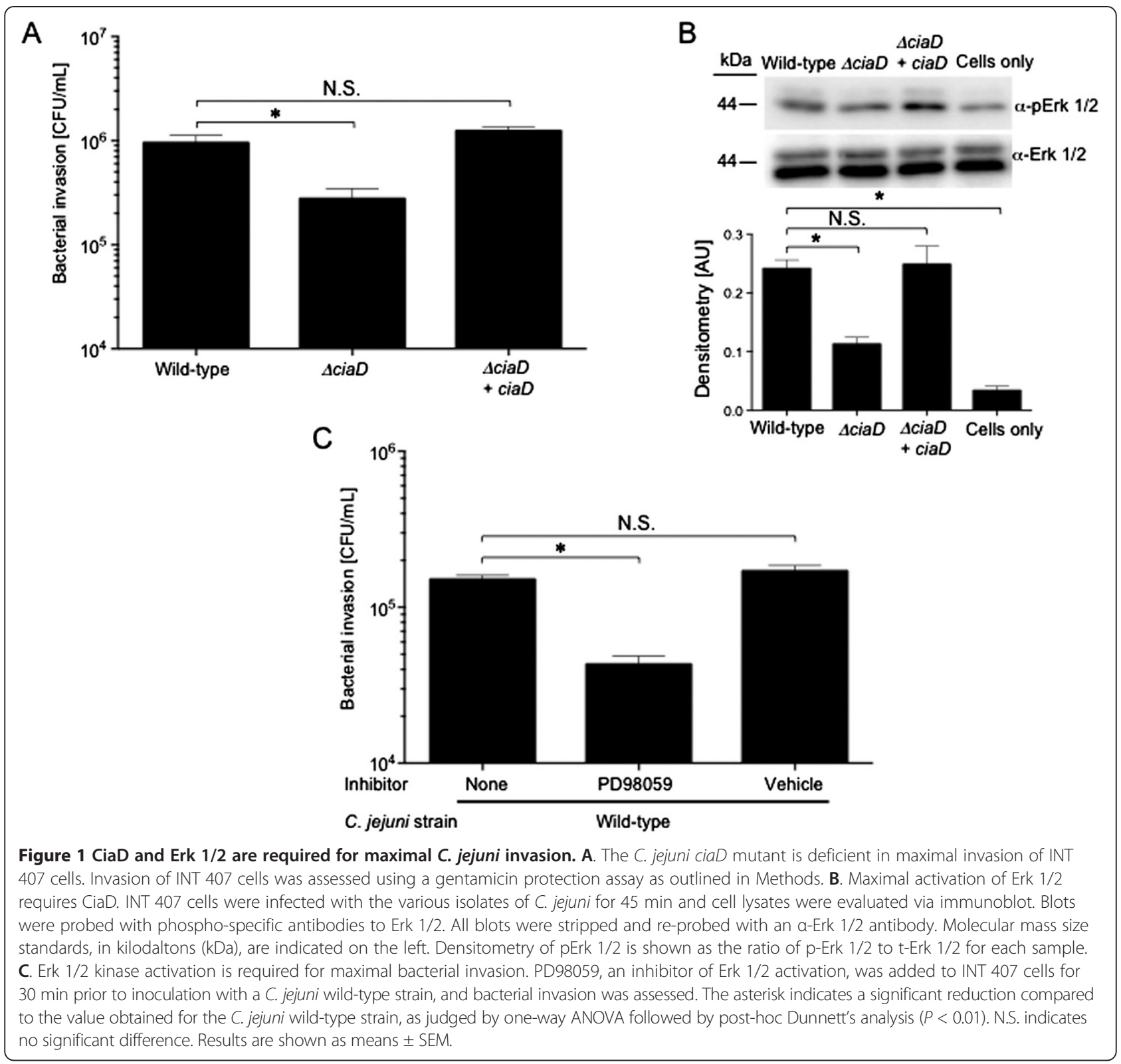

were performed to assess membrane ruffling of INT 407 cells upon infection with the $C$. jejuni wild-type strain, ciaD mutant, and ciaD mutant expressing a wild-type copy of ciaD in trans (ciaD complemented isolate), as well as cells infected with a $C$. jejuni wild-type strain that had been pretreated with the MEK 1/2 inhibitor PD98059 that blocks Erk 1/2 activation. Representative scanning electron microscopy (SEM) images of the various $C$. jejuni strains interacting with host INT 407 cells are shown in Figure 2A1-9. Both flagellated and non-flagellated bacteria were visualized bound to the host cells. The observation of non-flagellated bacteria bound to the cells was presumably due to the method of fixation, as all of the bacterial isolates were highly motile as judged by motility assays (not shown). We observed that $28.8 \% \pm 6.9 \%$ of untreated and uninfected INT 407 cells had membrane ruffling (Figure 2A1). In contrast, $57.9 \% \pm 5.7 \%$ of INT 407 cells had pronounced membrane ruffling when inoculated with a $C$. jejuni wild-type strain (Figure 2A2). Inoculation of INT 407 cells with the ciaD mutant resulted in membrane ruffling in $45.1 \% \pm 5.8 \%$ of the cells (Figure 2A3), whereas inoculation of cells with the ciaD complemented isolate resulted in membrane ruffling in $55.8 \% \pm 5.6 \%$ of the cells (Figure 2A4). Pretreatment of host cells with the Erk $1 / 2$ inhibitor reduced the percentage of host cells with membrane ruffling to $42.4 \% \pm 4.4 \%$ 


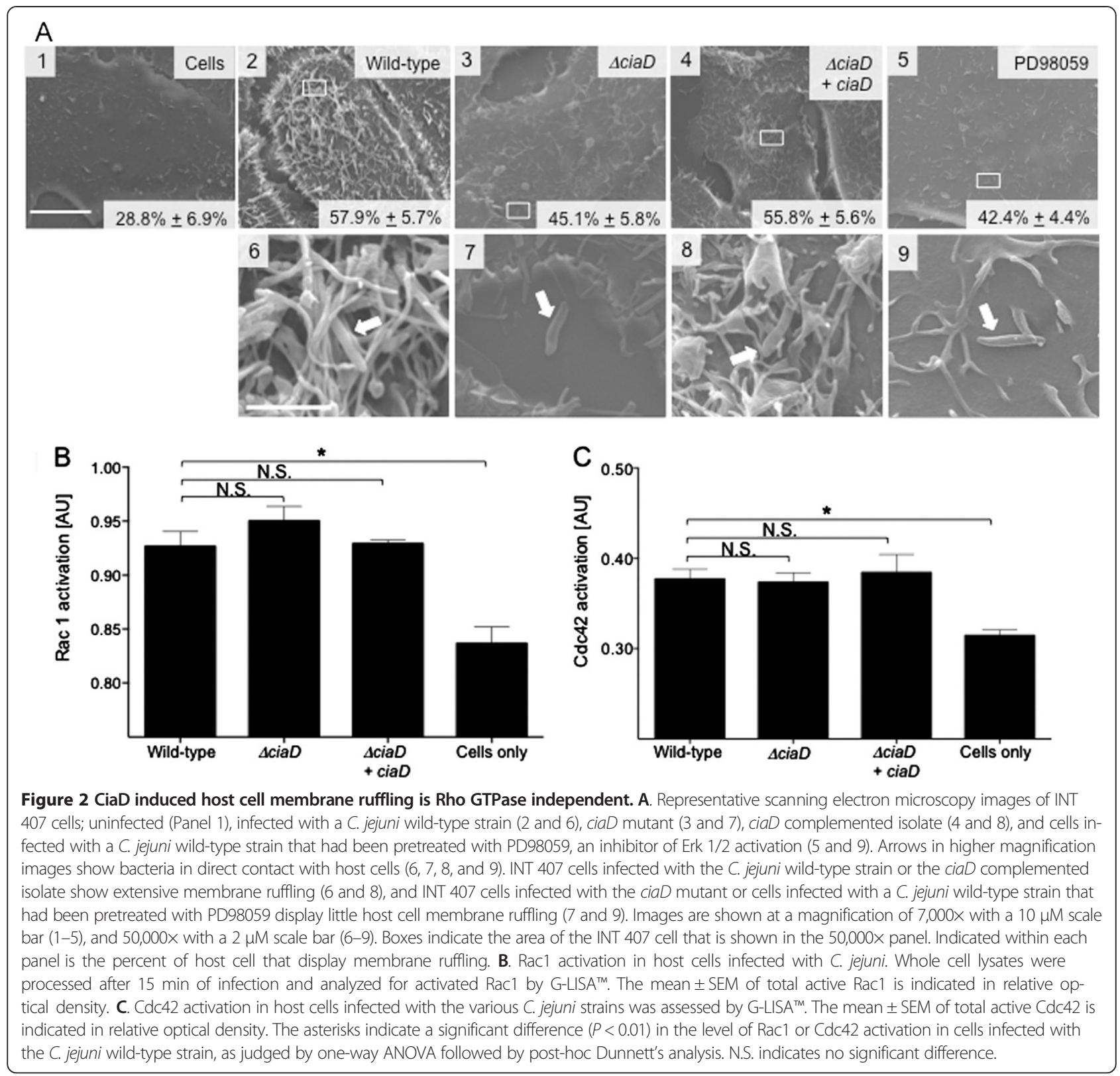

(Figure 2A5). Based on these data, we concluded that maximal membrane ruffling of host INT 407 cells requires CiaD and Erk $1 / 2$.

Given that the $C$. jejuni ciaD mutant was found to be deficient in stimulating membrane ruffling, we investigated whether there was a defect in Rho GTPase activation. The ciaD mutant exhibited normal Rac1 (Figure 2B) and Cdc42 (Figure 2C) activity when compared to the C. jejuni wild-type strain, as determined by G-LISA ${ }^{\mathrm{mm}}$. The fact that the activation levels of the Rho GTPases are not changing in the $C$. jejuni ciaD mutant was interesting, as there were clear reductions in bacterial invasion and host cell membrane ruffling. These data indicate that activated Rac1 and Cdc42 require assembly and/or activation of scaffold or accessory proteins to facilitate lamellipodia and filopodia extensions. Given the complexity of the $C$. jejuni-mediated invasion complex, we chose to focus on the role of CiaD-mediated Erk 1/2 activation and the potential targets of Erk $1 / 2$ that participate in membrane ruffling.

\section{CiaD mediated Erk 1/2 activation is required for cortactin serine phosphorylation}

Experiments were performed to determine if Erk 1/2 participates in transcriptional regulation of genes and/or activation of cytosolic signaling proteins necessary for actin 
cytoskeleton rearrangement, leading to $C$. jejuni host cell invasion and membrane ruffling. We first assessed the role of Erk $1 / 2$ mediated transcriptional regulation in C. jejuni invasion of host cells. To prevent Erk 1/2 mediated transcriptional activation in response to infection with C. jejuni, host INT 407 cells were pre-treated with 5,6dichloro-1-beta-D-ribofuranosylbenzimidazole (DRB). DRB inhibits Cdk-activating kinase (a TFIIH-associated kinase), thereby preventing transcription by RNA polymerase II [29]. To determine the concentration of DRB necessary to inhibit transcription, cells were pre-treated with different concentrations of DRB and the secretion of interleukin-8 (IL-8) from host cells was determined. The host cell chemokine IL-8 is transcribed, translated, and secreted from host cells in response to numerous bacterial pathogens, including C. jejuni [30-35]. Pretreatment of INT 407 cells with DRB resulted in a reduction in the amount of IL-8 in supernatants from $C$. jejuni infected cells (Figure 3A), suggesting that DRB effectively blocks the transcription of IL-8. However, pretreatment of INT 407 cells with DRB resulted in normal C. jejuni invasion of host cells (Figure 3B). These results suggest that Erk $1 / 2$ mediated transcriptional regulation is not involved in host cell actin cytoskeleton rearrangement necessary for $C$. jejuni host cell invasion.

Given that Erk 1/2 mediated transcriptional regulation is not required for cytoskeleton rearrangement, we performed experiments to determine if cytosolic signaling mediated by Erk 1/2 was altered or impaired. We chose to
A

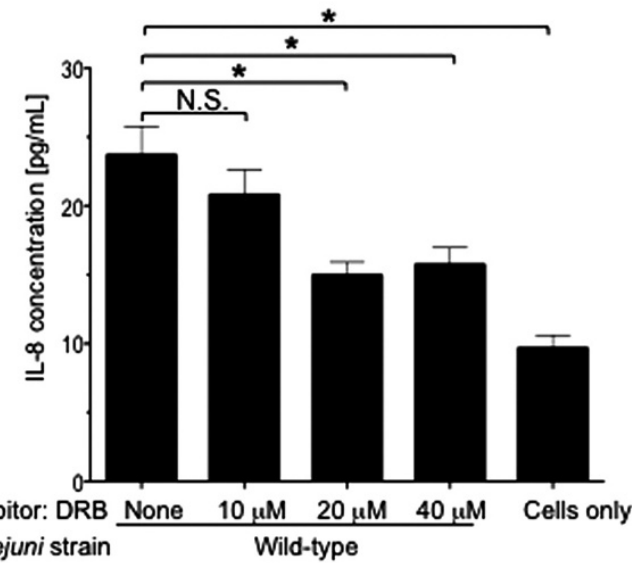

C. jejuni strain

B

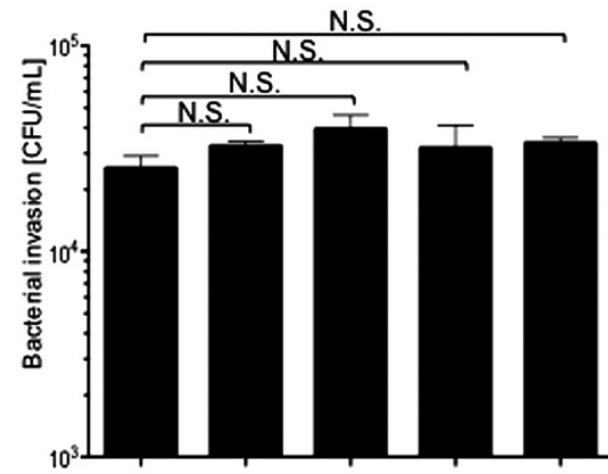

Inhibitor: DRB None $10 \mu \mathrm{M} \quad 20 \mu \mathrm{M} \quad 40 \mu \mathrm{M}$ Vehicle

C. jejuni strain
Wild-type

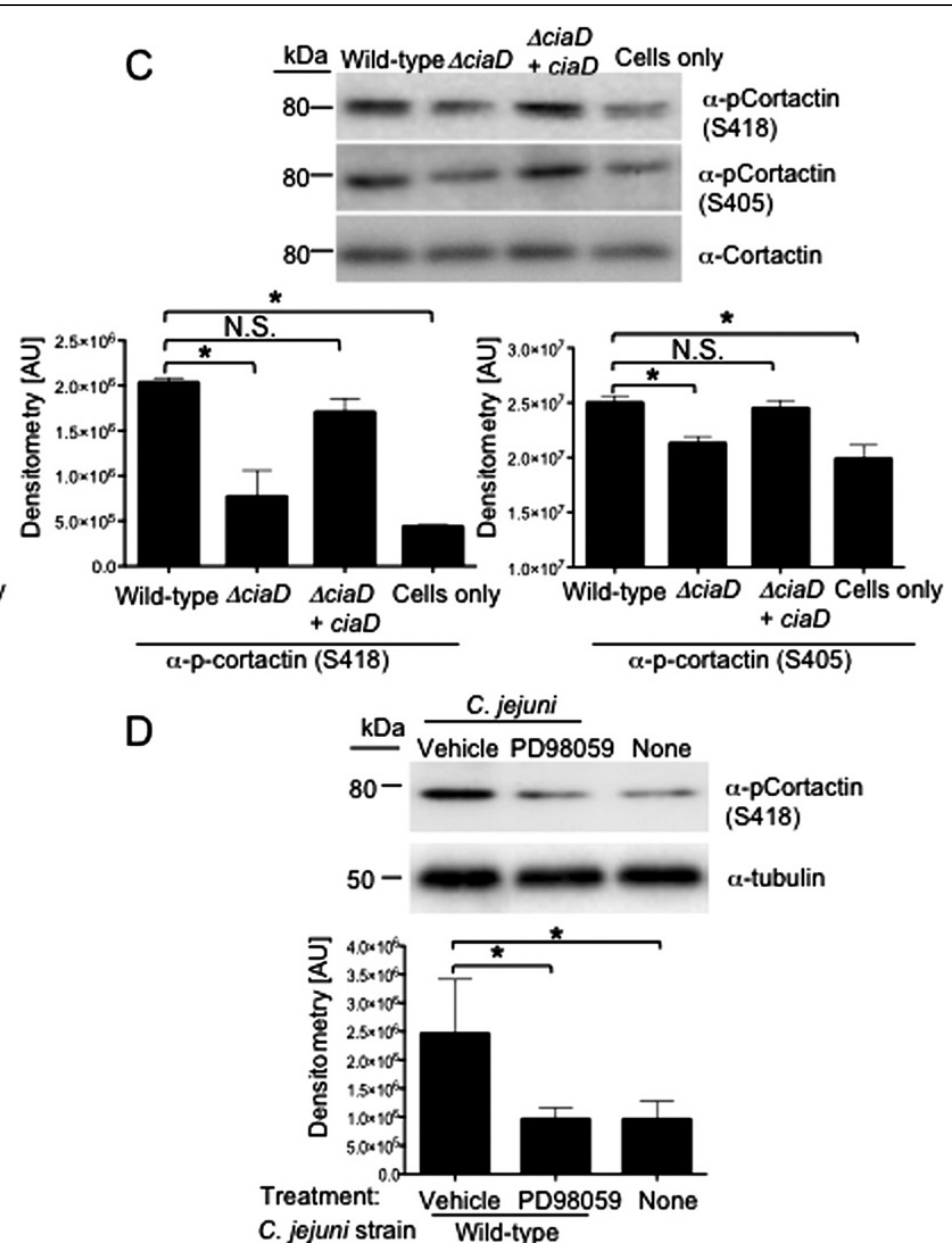

Figure 3 Erk $1 / 2$ is necessary for cytosolic signaling required for maximal C. jejuni invasion of host cell. A. INT 407 cells were infected with C. jejuni incubated for 6 h, and IL-8 quantified using an IL-8 ELISA. The transcription inhibitor 5,6-dichloro-1-beta-D-ribofuranosylbenzimidazole (DRB) was added to INT 407 cells for 30 min prior to infection with a C. jejuni wild-type strain. B. Transcription is not required for C. jejuni invasion. INT 407 cells were infected with C. jejuni and invasion was assessed. C. CiaD is required for serine phosphorylation of cortactin. INT 407 cells were infected with the various C. jejuni strains and cellular lysates were prepared. Blots were probed with phospho-specific antibodies to cortactin. The blot was stripped and re-probed with an a-cortactin antibody. Densitometry of p-cortactin is shown as the ratio of p-cortactin to total cortactin (t-cortactin) for each sample. D. Erk 1/2 is required for serine phosphorylation of cortactin. INT 407 cells were pre-treated with PD98059, an inhibitor of Erk 1/2 activation, and infected with a C. jejuni wild-type strain. Blots were probed with a phospho-specific antibody to cortactin. The blot was stripped and re-probed with an a-tubulin antibody. Molecular masses, in kilodaltons $(\mathrm{kDa})$, are indicated on the left. The asterisks indicate a significant difference $(P<0.01)$ compared to the value obtained for the C. jejuni wild-type strain, as judged by one-way ANOVA followed by post-hoc Dunnett's analysis. N.S. indicates no significant difference. 
investigate the Erk 1/2 mediated phosphorylation of the cytosolic actin binding protein cortactin, a known target of Erk $1 / 2$ and a component of the actin polymerization and nucleation complex [36]. In contrast to infection of INT 407 cells with a $C$. jejuni wild-type strain, the $C$. jejuni ciaD mutant was deficient in maximal phosphorylation of cortactin at the Erk $1 / 2$ phosphorylation sites S405 and S418, as judged by immunoblot analysis with the S418 and S405 phospho-specific antibodies to cortactin (Figure 3C). INT 407 cells infected with the C. jejuni ciaD complemented isolate restored the phosphorylation of cortactin to levels indistinguishable from infection with a C. jejuni wild-type strain (Figure $3 C$ ). This finding indicates that $\mathrm{CiaD}$ mediated activation of Erk 1/2 leads to the phosphorylation of cortactin on serine residues. Consistent with the fact that $\mathrm{CiaD}$ mediates Erk $1 / 2$ activation and Erk 1/2 mediates the phosphorylation of cortactin on S405 and S418, we found that pretreatment of INT 407 cells with the MEK 1/2 inhibitor PD98059 reduced phosphorylation of cortactin on S418 in response to C. jejuni infection, similar to the level observed in uninfected cells (Figure 3D). The inhibition of cortactin serine phosphorylation by treatment of cells with PD98059 is in agreement with published data [37]. However, this is the first report showing that cortactin becomes activated in response to C. jejuni infection. Given that $\mathrm{CiaD}$ is required for maximal cortactin activation, we assessed the role of cortactin phosphorylation in C. jejuni invasion of host cells.

\section{Cortactin serine phosphorylation is required for maximal invasion}

To determine if cortactin is required for $C$. jejuni invasion of host cells, we used small interfering RNA (siRNA) to knockdown cortactin and siRNA to knockdown the downstream complex protein N-WASP. N-WASP is a known component of the actin nucleation and polymerization complex and is necessary for the complete activation of Arp 2/3. More specifically, serine phosphorylation of cortactin leads to the recruitment of N-WASP, activation of Arp 2/3, and actin remodeling [36]. INT 407 cells were transfected with siRNA to cortactin or siRNA to N-WASP, and $C$. jejuni invasion of host cells was evaluated using the gentamicin protection assay. The knockdown of cortactin resulted in a significant reduction in the number of C. jejuni internalized by host cells (Figure 4A). The knockdown of N-WASP also significantly reduced the amount of internalized $C$. jejuni (Figure 4A). Effective knockdown of cortactin $(60.6 \% \pm 3.3 \%$ knockdown $)$ and N-WASP $(67.8 \% \pm 1.1 \%$ knockdown) was demonstrated by immunoblot analysis (Figure 4B). These data support the proposal that cortactin and N-WASP are necessary for maximal C. jejuni invasion of host cells.

To evaluate the specific contribution of Erk $1 / 2$ phosphorylation of cortactin at S405 and S418 in C. jejuni host cell invasion, phosphorylation null constructs of cortactin were utilized and the gentamicin protection assay was performed. INT 407 cells were transfected with cortactin-EGFP phosphorylation null constructs with the following mutations: S405A, S418A, and S405/ 418A (double serine mutant). The contributions of c-Src (tyrosine) phosphorylation of cortactin were also evaluated, as c-Src phosphorylation of cortactin is known to be important for the invasion of other pathogens [2,3,5]. INT 407 cells were transfected with cortactin-EGFP Y421F and Y421/470/486 F (triple tyrosine mutant) mutant constructs to evaluate the role of c-Src phosphorylation of cortactin. We found that both cortactin serine and tyrosine phosphorylation are required for maximal invasion of host cells by $C$. jejuni, as judged by the gentamicin protection assay (Figure 4C). Equal expression of the cortactin-EGFP phosphorylation null constructs was confirmed via immunoblot analysis (Figure 4D). In support of the finding that tyrosine phosphorylation of cortactin is required for C. jejuni invasion, inhibition of the upstream kinase c-Src with the inhibitor PP2 prevented C. jejuni internalization (Additional file 1: Figure S1). This is the first report to our knowledge demonstrating that serine phosphorylation of cortactin by Erk $1 / 2$ and tyrosine phosphorylation of cortactin by c-Src are required for $C$. jejuni invasion of host cells. Based on these results, we hypothesized that cortactin and the serine phosphorylation of cortactin are necessary for $C$. jejuniinduced membrane ruffling.

\section{Cortactin serine phosphorylation is required for host cell membrane ruffling}

To evaluate the role of cortactin activation by $\mathrm{CiaD}$ in C. jejuni-mediated host cell membrane ruffling, we utilized EGFP-tagged cortactin to visualize membrane ruffling. INT 407 cells, which had been transfected with a cortactin-EGFP construct, were infected with the C. jejuni wild-type strain, ciaD mutant, and the ciaD complemented isolate and the cells examined by confocal microscopy. Uninfected cells transfected with cortactinEGFP exhibited diffuse cortactin localization with no distinct membrane ruffling of cell borders (Additional file 2: Figure S2 A-D). However, infection with the C. jejuni wild-type stain resulted in membrane ruffling (Additional file 2: Figure S2 E-H). In contrast, the C. jejuni ciaD mutant was deficient in membrane ruffling and exhibited diffuse cortactin localization similar to that of uninfected cells (Additional file 2: Figure S2 I-L). Host cell membrane ruffling was restored when cells were infected with the C. jejuni ciaD complemented isolate (Additional file 2: Figure S2 M-P). While this experiment indicated that CiaD is necessary for $C$. jejuni-induced membrane ruffling, it was not clear if cortactin is required for membrane ruffling. 


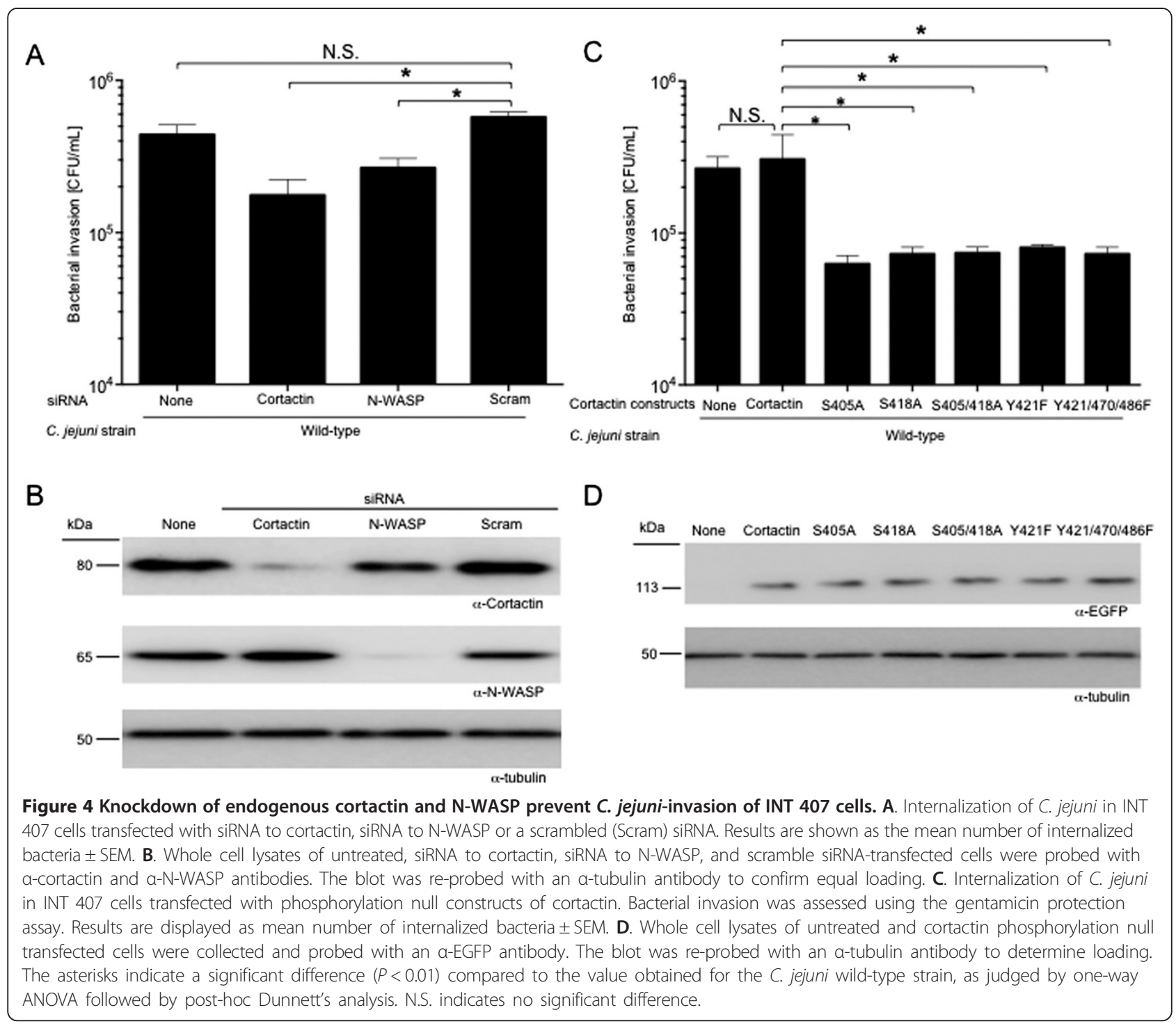

Experiments were performed to determine if serine phosphorylation of cortactin is necessary for C. jejuniinduced membrane ruffling. INT 407 cells, which had been transfected with cortactin-EGFP S405A, S418A, and S405/418A phosphorylation null constructs, were infected with a $C$. jejuni wild-type strain and evaluated by confocal microscopy. Uninfected cells transfected with cortactinEGFP exhibited diffuse cortactin localization with no distinct membrane ruffling of the cell borders (Figure 5A-D). Infection of cortactin-EGFP transfected cells with the C. jejuni wild-type strain resulted in distinct membrane ruffling (Figure 5E-H). C. jejuni infection of INT 407 cells transfected with EGFP-cortactin S405A (Figure 5I-L), S418A (Figure 5M-P), and S405/418A (Figure 5Q-T) phosphorylation null constructs resulted in a diffuse localization of cortactin and no observable membrane ruffling in response to $C$. jejuni was observed. These results indicate that cortactin is required for C. jejuni-induced membrane ruffling.

Scanning electron microscopy was performed to determine the extent of $C$. jejuni-induced membrane ruffling in cells transfected with siRNA to cortactin and siRNA to N-WASP. More specifically, INT 407 cells were transfected with a scrambled siRNA, siRNA to cortactin, or siRNA to N-WASP, and infected with a C. jejuni wild-type strain. INT 407 cells were also transfected with cortactin-EGFP S405A, S418A, and S405/ 418A phosphorylation null constructs. Representative images of $C$. jejuni interaction with host cells are shown in Figure 6A-P. We observed that $23.9 \% \pm 8.6 \%$ of untreated and uninfected INT 407 cells had membrane ruffling (Figure 6A). Membrane ruffling was observed in $69.6 \% \pm 7.1 \%$ of the cells infected with a $C$. jejuni wildtype strain (Figure 6B). Treatment of INT 407 cells with 


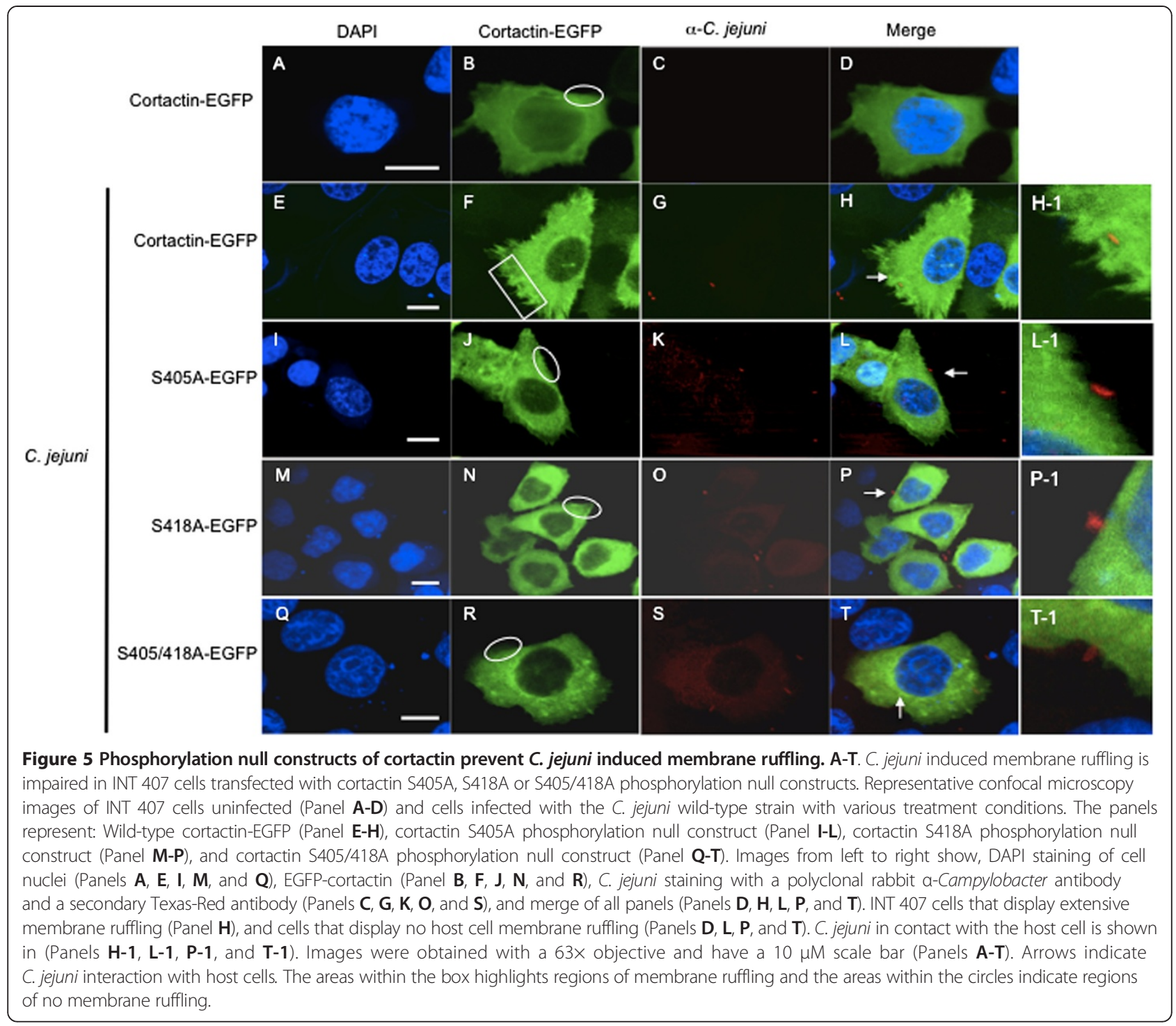

a non-coding scrambled siRNA prior to infection with C. jejuni resulted in levels of membrane ruffling similar to untreated cells infected with C. jejuni (Figure 6C). Treatment of host cells with siRNA to N-WASP and siRNA to cortactin reduced membrane ruffling to $26.8 \% \pm$ $6.0 \%$ and $27.9 \% \pm 6.4 \%$, respectively (Figure $6 \mathrm{D}$ and $\mathrm{E}$ ). We also observed a significant decrease in host cell membrane ruffling when cells were transfected with phosphorylation null constructs of cortactin (Figure 6F-H). Specifically, the cortactin-EGFP S405A, S418A, and S405/418A phosphorylation null constructs reduced membrane ruffling to $25.4 \% \pm 4.2 \%, 28.6 \% \pm 5.8 \%$, and $31.5 \% \pm 3.9 \%$, respectively. Noteworthy is that treatment of INT 407 cells with siRNA or phosphorylation null constructs prevented membrane ruffling from occurring even in the presence of direct bacterial contact (compare panels J and $\mathrm{K}$ to panels L-P). These data indicate that cortactin and N-WASP are required for maximal membrane ruffling induced by $C$. jejuni.

\section{Cortactin, Erk 1/2, and N-WASP complex formation is CiaD dependent}

Immunoblot and immunoprecipitation (IP) experiments were performed to determine if cortactin and Erk $1 / 2$ are associated in response to $C$. jejuni infection. INT 407 cells were infected with $C$. jejuni for 45 minutes and cortactin was precipitated using an $\alpha$-cortactin antibody. Infection of INT 407 cells with a $C$. jejuni wild-type strain resulted in a significant increase (above uninfected cells) in the amount of phosphorylated (activated) cortactin (Figure 7). In contrast, the amount of activated cortactin significantly decreased in INT 407 cells infected with the C. jejuni ciaD mutant. Specifically, infection with the $C$. jejuni wild-type strain and the ciaD complemented 


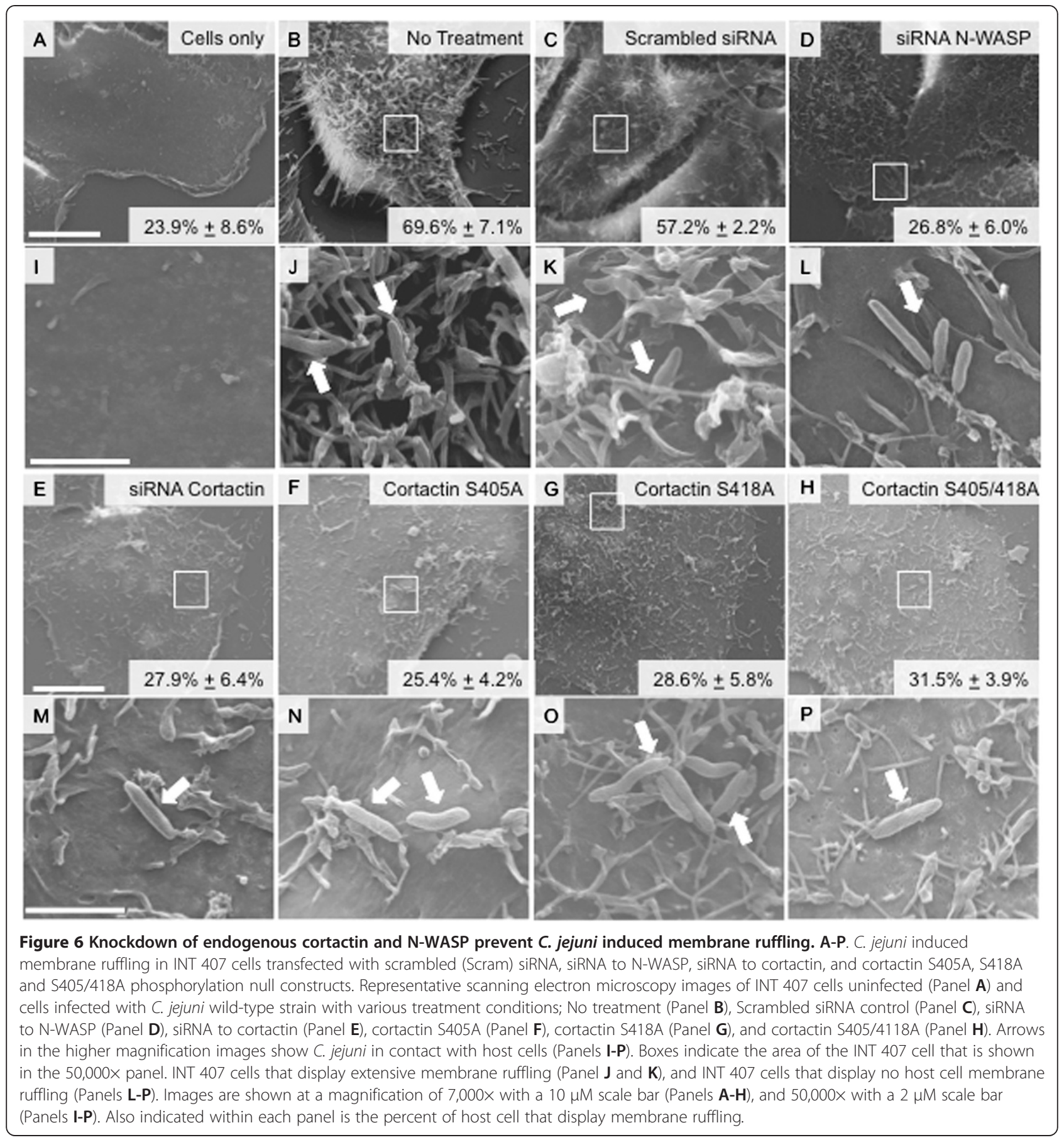

isolate resulted in a significant increase in the level of activated cortactin, but the $C$. jejuni ciaD mutant was indistinguishable from uninfected cells (Figure 7). The association of Erk $1 / 2$ and cortactin in cells infected with the $C$. jejuni wild-type strain, ciaD mutant, and ciaD complemented isolate was also determined by immunoprecipitation. We found that $\mathrm{CiaD}$ is required for the maximal association of cortactin with phosphorylated
Erk 1/2 (Figure 7). The association of N-WASP with cortactin was also determined to occur in a CiaD dependent manner (Figure 7). This finding suggests that N-WASP is associated with the serine phosphorylated form of cortactin. Others have shown that serine phosphorylation of cortactin is necessary for N-WASP association [36,38-40]. The IP experiments performed also revealed that cortactin associates with phospho-Erk $1 / 2$ and N- 


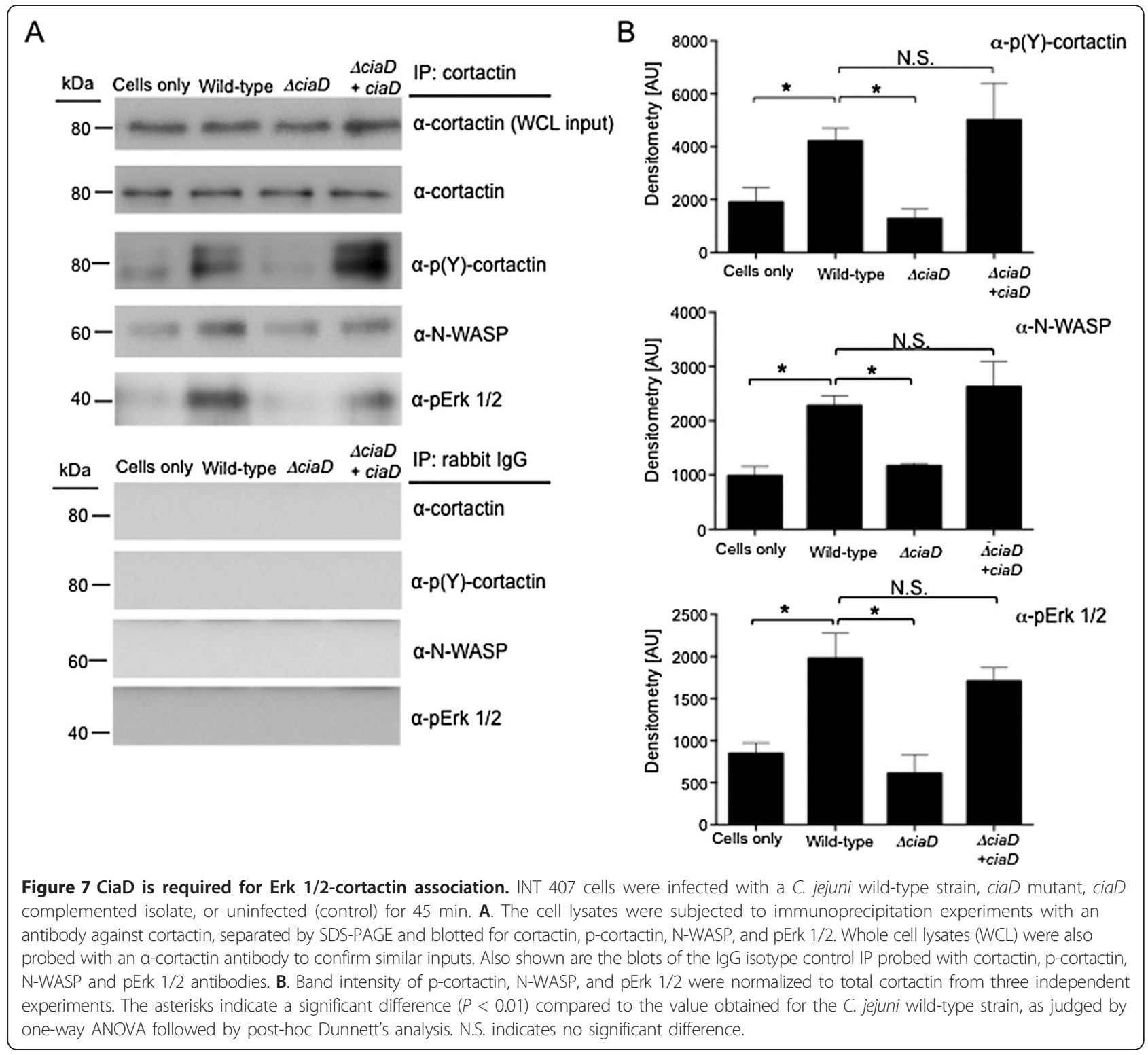

WASP upon C. jejuni infection, and that N-WASP and phospho-Erk $1 / 2$ association with cortactin is dependent on $\mathrm{CiaD}$ (Figure 7). These data show that $C$. jejuni induces the formation of the actin nucleation and polymerization complex Erk 1/2-cortactin-N-WASP, and that this association is, in part, dependent of the $C$. jejuni effector protein CiaD. These data also show that the recruitment of N-WASP to cortactin requires Erk 1/2 serine phosphorylation of cortactin.

\section{Discussion}

This study was performed to further elucidate the mechanism of $C$. jejuni invasion of host cells. More specifically, we investigated the role of Erk $1 / 2$ and cortactin in C. jejuni invasion of host cells. Erk $1 / 2$ is a serine/threonine kinase that is part of the Ras-Raf-MEK-ERK signal transduction cascade. Erk $1 / 2$ is activated by dual phosphorylation at Y204/187 and T202/185 catalyzed by MEK 1/2 [41,42]. Erk $1 / 2$ catalyzes the phosphorylation of hundreds of cytoplasmic and nuclear proteins and participates in numerous cellular processes including cell adhesion, cell cycle progression, cell migration, cell survival, differentiation, metabolism, proliferation, and transcription [42]. Cortactin is a filamentous actin binding protein that is a crucial link between the organization of structural proteins, such as actin, and cellular signal transduction pathways. Cortactin stimulates actin polymerization via interaction with N-WASP through its SH3 domain, and binding of Arp 2/3 through its N-terminal domain [36]. Cortactin is regulated by phosphorylation of Y421, Y470, and Y486 by c-Src and other tyrosine kinases [36]. Likewise, Erk 1/2 phosphorylates S405 and S418 
of cortactin $[36,38]$. There is also evidence that PAK phosphorylates cortactin, however the implications of PAK serine phosphorylation are poorly defined $[1,36]$. Work by Martinez-Quiles et al. (2004) revealed that phosphorylation of cortactin by Erk $1 / 2$ acts as a positive regulatory event and Src phosphorylation acts as a negative regulatory event in actin cytoskeletal rearrangement by activation/deactivation of N-WASP and Arp2/3 [40]. Additionally, Kelley et al. [39] demonstrated that concurrent phosphorylation of cortactin by Erk $1 / 2$ and tyrosine kinases allow cells to regulate actin dynamics through $\mathrm{N}$ WASP. Taken together, it is clear that the activation and deactivation of cortactin by phosphorylation is a dynamic process. In the present study, we showed that phosphorylation of cortactin on S405, S418, Y421, Y470, and Y486 are required for maximal invasion of host cells by $C$. jejuni. Specifically, we show that CiaD is required for maximal activation of Erk 1/2 (Figure 1). Activation of Erk 1/2 leads to the phosphorylation of S405 and S418 on cortactin (Figure 3). Also, the association of cortactin with Erk $1 / 2$ is dependent on CiaD (Figure 7). Furthermore, we found that serine phosphorylation of cortactin is required for maximal $C$. jejuni induced host cell membrane ruffling. These findings provide the basis for a detailed model of $C$. jejuni invasion of host cells (Figure 8).

Previous work has shown that Dock180 and its binding partner ELMO (Engulfment and Cell Motility) form a bipartite guanine nucleotide exchange factor (GEF), resulting in the activation of Rac1 and membrane ruffling [27]. C. jejuni invasion of cells is also accompanied by the activation of Cdc42 [28,45]. Interestingly, several effector proteins from Salmonella enterica, including SopE, SopE2 and SopB, modulate the activity of Cdc42 and Rac1 to manipulate actin cytoskeleton rearrangements $[48,49]$. Noteworthy is that the IcsA effector protein from Shigella flexneri promotes filopodia formation by binding and activating N-WASP in a Cdc42-like fashion [50]. To determine the role of $\mathrm{CiaD}$ in C. jejuni invasion of host cells, we first evaluated the Rho GTPases Rac1 and Cdc42 as a loss in the activation of either

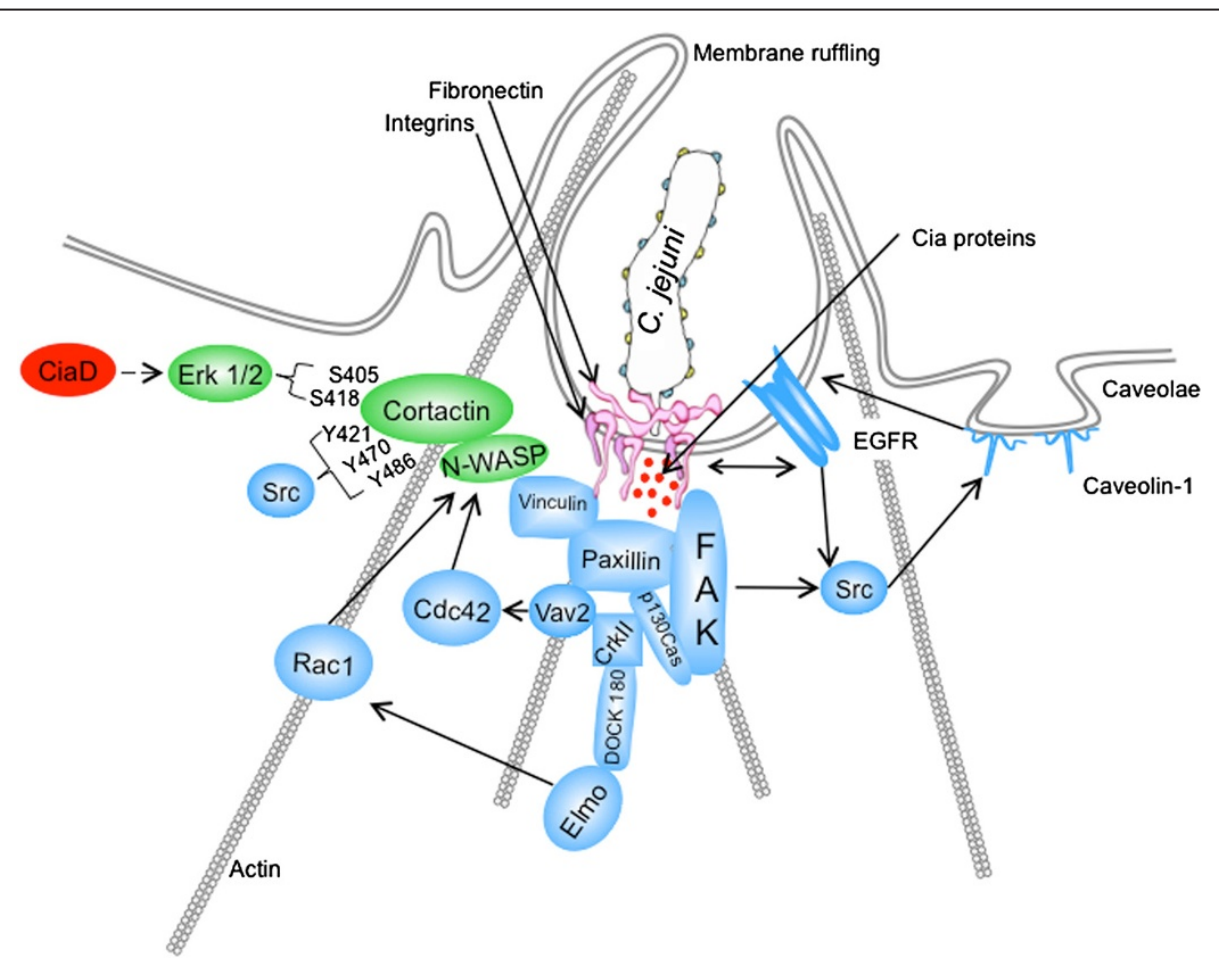

Figure 8 Model of $C$. jejuni internalization. C. jejuni invasion of host cells. Step 1: C. jejuni binds to fibronectin (Fn) via the two C. jejuni Fn binding proteins CadF (blue dots) and FlpA (yellow dots) $[43,44]$ causing activation of the $a_{5} \beta_{1}$ integrin receptors and the epidermal growth factor receptor (EGFR) $[27,45]$. Step 2: Activation of the $a_{5} \beta_{1}$ integrin leads to the recruitment and partial activation of FAK and paxillin $[27,46]$. Step 3: The delivery of the Campylobacter invasion antigens (e.9., CiaD shown in red) to the host cell $[16,19,27,47]$ leads to the maximal activation of key components of the focal complex (i.e., FAK, paxillin, vinculin, p130Cas, Src, and the Crkll/DOCK-180/ELMO complex) [27,28,45, Konkel et. al, Invasion of epithelial cells by Campylobacter jejuni is independent of caveolin-1, In Submission]. Step 4: Focal complex activation, in conjunction with CiaD, leads to the phosphorylation of Erk 1/2. Caveolin-1, Vav2, Rac1, and Cdc42 are also activated following focal complex activation [27,28,45]. Step 5: Activation of Erk 1/2 and Src leads to the phosphorylation of cortactin, which allows for the Rho GTPases Rac1 and Cdc42 to activate N-WASP associated with phosphorylated cortactin, promoting actin cytoskeletal reorganization. Highlighted in this model is the role of CiaD in C. jejuni internalization. Specifically, CiaD is necessary for the maximal activation of the Erk $1 / 2$ and cortactin signaling pathways. Components of the focal complex and focal complex associated proteins are shown in blue. The newly identified components of the C. jejuni invasion complex are shown in green. 
protein could explain the invasion deficiency of the ciaD mutant. INT 407 cells infected with the C. jejuni ciaD mutant exhibited levels of Rho GTPase activation similar to that of cells infected with the $C$. jejuni wild-type strain. This is in stark contrast to cells infected with a $C$. jejuni ciaC mutant that display a significant reduction in Rac1 activation [27]. The reduction in Rac1 activity with the $C$. jejuni ciaC mutant is in agreement with the fact that there are fewer sites of co-localized Rac1 in INT 407 cells infected with the $C$. jejuni ciaC mutant versus a $C$. jejuni wild-type strain [27]. Our data supports the proposal that $\mathrm{CiaD}$ is manipulating cellular signaling cascades and altering actin nucleation at a site downstream from Rac1 and Cdc42. Moreover, our results indicate that $\mathrm{CiaC}$ and $\mathrm{CiaD}$ manipulate at least two distinct host cell targets that are necessary for $C$. jejuni invasion of host cells.

Based on the observation that Erk $1 / 2$ is critical for C. jejuni invasion of host cells, we performed experiments to determine if: 1) Erk $1 / 2$ is transcriptionally regulating cellular components involved in cell invasion; and/or 2) Erk $1 / 2$ is necessary for the activation of cytosolic cellular signaling cascades involved in cytoskeleton rearrangement. We found that the transcription of the gene that encodes for IL- 8 is not required for invasion, but that Erk $1 / 2$ is required for the serine phosphorylation of cortactin. As previously stated, cortactin is an actin-binding protein that recruits N-WASP and activates Arp 2/3, leading to actin remodeling [36]. Interestingly, Erk $1 / 2$ activation stimulates bacterial capture of Shigella by filopodia [51], while the OspF effector protein from Shigella harbors phosphatase activity to inactivate mitogen-activated protein kinases (MAPKs), including Erk 1/2, c-Jun N-terminal kinase, and p38, post-invasion [52]. Collectively, these data highlight the fact that Erk $1 / 2$ is a key component of the $C$. jejuni invasion complex and that bacterial pathogens can manipulate membrane extensions (e.g., lamellipodia and filopodia) by targeting Erk $1 / 2$.

Cortactin is likely involved in the uptake of pathogenic bacteria into host cells, as it acts in concert with N-WASP to activate the Arp2/3 complex. It is plausible for a pathogen to activate cortactin directly or to activate cortactin indirectly via Erk $1 / 2$ or Src. For example, the IpaC effector protein from Shigella mediates Srcdependent phosphorylation of cortactin, thereby promoting actin polymerization [53]. We found that serine phosphorylation of cortactin by $C$. jejuni is dependent upon Erk 1/2, as the level of phospho-cortactin in C. jejuni infected cells treated with the PD98059 inhibitor was indistinguishable from uninfected cells.

Here we demonstrate that the formation of the Erk 1/2cortactin-N-WASP complex is dependent on the C. jejuni effector protein $\mathrm{CiaD}$. To our knowledge this is the first report of the involvement of cortactin and N-WASP in host cell invasion by $C$. jejuni. In addition, this is the first report showing that Erk 1/2 mediated serine phosphorylation of cortactin is required for $C$. jejuni invasion of host cells. Future studies will focus on the identification of the direct target of $\mathrm{CiaD}$ and the kinetics and regulation of cortactin in bacterial invasion. This work provides new insight into C. jejuni pathogenesis and the complex signaling events exploited by bacterial pathogens during the process of invasion. Equally important, it contributes to the understanding of the phosphorylation of cortactin in bacterial infection.

\section{Conclusion}

In this study, we present a model for $C$. jejuni invasion of host cells (Figure 8). We show that C. jejuni activates Erk 1/2. Specifically, we found that Erk $1 / 2$ activation is dependent on the $C$. jejuni effector protein CiaD. Similarly, we found that Erk $1 / 2$ activation is necessary for bacterial invasion because it is necessary for the phosphorylation of serine residues in cortactin. Moreover, inhibition of serine phosphorylation results in decreased bacterial invasion and host cell membrane ruffling. The requirement of serine phosphorylation of cortactin by Erk $1 / 2$ in C. jejuni host cell invasion represents a mechanistic basis for how Erk 1/2 inhibition leads to impaired C. jejuni invasion.

\section{Methods}

\section{Bacterial strains, tissues culture types}

The Campylobacter jejuni F38011 clinical isolate was used in this study. All $C$. jejuni isolates were cultured on Muller-Hinton agar plates containing bovine citrated blood $(\mathrm{MHB})$ with the appropriate antibiotic at the following final concentrations: chloramphenicol 8 microgram $/ \mathrm{mL}$ and tetracycline 2 microgram $/ \mathrm{mL}$. Cultures were grown at $37^{\circ}$ $\mathrm{C}$ in a microaerobic chamber $\left(5 \% \mathrm{O}_{2}, 10 \% \mathrm{CO}_{2}\right.$, and $85 \%$ $\mathrm{N}_{2}$ ). INT 407 cells (ATCC CCL 6) were obtained form the American Type Culture Collection (ATCC). INT 407 cells were cultured in minimal essential medium (MEM) supplemented with $10 \mathrm{mM}$ sodium pyruvate, $20 \mathrm{mM}$ glutamine, and $10 \%(\mathrm{v} / \mathrm{v})$ fetal bovine serum (FBS).

\section{Preparation of INT 407 whole cell lysates}

Whole cell lysates (WCL) of INT 407 cells were prepared by the addition of lysis buffer, as described previously [27]. The lysates were collected and analyzed by SDS-PAGE coupled with immunoblot analyses. The protein concentration of each sample was determined by the bicinchoninic acid (BCA, Pierce, Rockford, IL) protein assay and normalized prior to SDS-PAGE.

\section{Immunoblot analysis, cellular inhibitors, antibodies, and densitometry analysis}

Immunoblots were performed as described previously [27]. INT 407 cell lysates were collected and analyzed by 
SDS-polyacrylamide gel electrophoresis. The proteins were transferred to a polyvinylidene fluoride (PVDF) membrane, and probed with the indicated antibodies. Primary antibodies reactive against N-WASP (Cell Signaling Technology, Cat \# 4848), phospho-Erk 1/2 (Cell Signaling Technology, Cat \# 4377), total-Erk 1/2 (Santa Cruz, Dallas, TX, Cat \# sc-94) cortactin (Cell Signaling Technology, Cat \# 3503), phospho-cortactin (S418) (Protea, Morgantown, WV, Cat \# AB-110), phospho-cortactin (S405) (Protea Biosciences, Inc., Morgantown, WV, Cat \# AB-100), tyrosine-phospho-cortactin (Millipore, Billerica, MA, Cat \# 05-180) and tubulin (Sigma, Cat \# T6199) were used at a 1:1000 dilution and incubated overnight at $4^{\circ} \mathrm{C}$. The $\alpha$-rabbit IgG or the $\alpha$-mouse IgG (Sigma, St. Louis, MO) secondary antibodies were applied at a 1:2000 dilution for $1 \mathrm{~h}$ at room temperature. Band intensity was quantified using a LAS 4000 mini (GE healthcare) and the Multi Gauge V3.0 (Fujifilm, Valhalla, NY) software package. Densitometry analysis is shown as the ratio of phosphoprotein to total protein (i.e., p-Erk 1/2 to total Erk $1 / 2$ and p-cortactin to total cortactin) or as the ratio of target protein to cortactin in the IP experiments (i.e., NWASP to total cortactin and p-Erk $1 / 2$ to total cortactin). Inhibitors or vehicle [dimethyl sulfoxide (DMSO)] were added to INT 407 cells $30 \mathrm{~min}$ prior to infection and maintained throughout the assay. The Erk 1/2 inhibitor PD98059 (Selleck, Houston, TX, Cat \# S1177) was used at $50 \mu \mathrm{M}$. The c-Src inhibitor PP2 (Sigma, Cat \# P0042) was used at $5,10,20$, and $40 \mu \mathrm{g} / \mathrm{mL}$. The transcription inhibitor 5,6-dichloro-1-beta-D-ribofuranosylbenzimidazole (DRB) (Sigma, Cat \# D1916) was used at 10, 20, and $40 \mu \mathrm{M}$. Cell death was quantified with trypan blue staining. No significant death of INT 407 cells was observed with any of the treatments (not shown).

\section{Binding and internalization assays}

Binding and internalization assays were performed as described elsewhere [27]. INT 407 cells were seeded at a density of $1.5 \times 10^{5}$ into 24-well flat bottom tissue culture trays (BD Falcon, Franklin Lakes, NJ, Cat \# 353047). Bacteria were suspended in MEM containing 1\% FBS and added to cells at a multiplicity of infection (MOI) of 100. Trays were centrifuged at $800 \times g$ to promote bacterial cell contact. Cells were lysed with $0.1 \%$ Triton-X100 and plated onto MHB agar for bacterial enumeration. C. jejuni host cell invasion was assessed by lysing INT 407 cells and enumerating the internalized bacteria following a $3 \mathrm{~h}$ incubation with 250 microgram $/ \mathrm{mL}$ of gentamicin.

\section{Transfection of phosphorylation null constructs}

Human Cortactin-GFP and S405A, S418A, S405/S418A, Y421F, and Y421/470/486F phosphorylation null GFP constructs of cortactin were generously provided by Dr. Scott Weed from West Virginia University [38].
Plasmids were purified using the Qiagen Plasmid Purification Kit (Qiagen, Valencia, CA) according to the manufacturer's protocols. Purified plasmids were quantified using NanoDrop 2000c (Thermo Scientific, Wilmington, DE) and normalized to $200 \mathrm{ng} / \mu \mathrm{l}$. Purified plasmids where transfected into INT 407 cells seeded on glass coverslips at $3 \times 10^{5}$. Transfections where performed using the Qiagen Effectene Transfection reagent (Qiagen, Valencia, $\mathrm{CA})$, according to the manufacturer's specifications.

\section{Confocal microscopy}

INT 407 cells were infected with $C$. jejuni for $45 \mathrm{~min}$ at $37^{\circ} \mathrm{C}$ in a $5 \% \mathrm{CO}_{2}$ incubator prior to fixation with $3.7 \%$ paraformaldehyde for $15 \mathrm{~min}$. C. jejuni were stained with a $1^{\circ}$ rabbit $\alpha-C$. jejuni antibody (Konkel Laboratory Collection) and a $2^{\circ}$ Texas Red dye-conjugated donkey $\alpha$-rabbit antibody (Jackson ImmunoResearch Labs, West Groves, PA). The coverslips were mounted with VectaShield and 4',6-diamidino-2-phenylindole (DAPI, Vector Laboratories, Burlingame, CA) added to stain DNA. Images were obtained using a Zeiss confocal microscope using a 63X, 1.4 NA water immersion objective lens.

\section{Scanning electron microscopy}

Scanning electron microscopy was performed as described elsewhere [27]. INT 407 cells were transfected with the phosphorylation null or siRNA constructs. C. jejuni was added to cells for 15 minutes. Quantification of membrane ruffling was done by two independent observers and tabulated. Cells were counted and cells positive for membrane ruffling were scored.

\section{Treatment of cells with small interfering RNA (siRNA)}

INT 407 cells were transfected with siRNA using lipofectamine RNAiMAX (Invitrogen, Grand Island, NY) according to the manufacturer's instructions. Cortactin stealth siRNA (Invitrogen, Grand Island, NY, Cat \# S4665), N-WASP stealth siRNA (Invitrogen, Cat \# S17132), and scrambled control siRNA (Invitrogen, Cat \# 46-2000) were applied to the cells $24 \mathrm{~h}$ prior to infection. Knockdown of endogenous proteins were confirmed by immunoblot.

\section{Assessment of Rho GTPase Rac1 and Cdc42 activation}

INT 407 cells were seeded into 6-well tissue culture trays at a density of $2 \times 10^{5}$ cells/well and serum starved for 24 h. C. jejuni was resuspended in PBS and added to the cells. The amount of activated Rac1 and Cdc42 in C. jejuni-infected and uninfected cells was determined using the G-LISA ${ }^{\mathrm{Tm}}$ Rac1 and Cdc42 Activation Assays according to the manufacturer's instructions (Cytoskeleton, Denver, CO). 


\section{Immunoprecipitation}

INT 407 cells were seeded at $3 \times 10^{6}$ cells per dish and serum starved in MEM for $3 \mathrm{~h}$ prior to the addition of C. jejuni or the uninfected (negative control). Forty-five min post-infection, cells were collected in ice-cold lysis buffer as described previously [27]. Immunoprecipitations were performed by incubating cell lysates with an $\alpha$-cortactin antibody (Cell Signaling Technology, Inc., Danvers, MA) at $4^{\circ} \mathrm{C}$ overnight and then adding protein $\mathrm{A} / \mathrm{G}$ beads at $4^{\circ} \mathrm{C}$ for $1 \mathrm{~h}$ with rotation. The bead complexes were washed and dissolved in sample buffer.

\section{IL-8 quantification}

Interleukin-8 levels in cellular supernatants were quantified with a commercial ELISA (OptEIA Set, Becton Dickinson, Cowley, Oxford, UK) using the manufacturer's protocol. INT 407 cells were pre-treated with DRB as described above and inoculated with $C$. jejuni. Cells were centrifuged for $5 \mathrm{~min}$ at $800 \times g$ to promote cell contact. The cells were incubated at $37^{\circ} \mathrm{C}$ for $6 \mathrm{~h}$ and the media were collected from each well. Supernatants were used immediately or frozen at $-20^{\circ} \mathrm{C}$.

\section{Statistical analysis}

All data were evaluated using one-way ANOVA followed by post-hoc Tukey's or Dunnet's analysis of the means, using Prism 6 (GraphPad Software, La Jolla, CA). Statistical significance was defined by a maximum value of $* \mathrm{P}<0.05$. All experiments were performed a minimum of three times to ensure reproducibility.

\section{Additional files}

Additional file 1: Figure S1. Inhibition of c-Src prevents $C$. jejuni invasion of INT 407 cells. Internalization of C. jejuni by INT 407 cells treated or untreated (control) with the c-Src inhibitor PP2 for 30 min prior to bacterial invasion. Bars represent the mean number of internalized bacteria \pm SEM. The asterisks indicate a significant difference $(P<0.01)$ compared to the value obtained for the C. jejuni wild-type strain, as judged by one-way ANOVA followed by post-hoc Dunnett's analysis.

Additional file 2: Figure S2. CiaD is required for membrane ruffling. A-P. CiaD is required for C. jejuni induced membrane ruffling in INT 407 cells transfected with cortactin-EGFP. Representative confocal microscopy images of INT 407 cells uninfected (Panel A-D) and infected with a C. jejuni wild-type strain (Panel E-H), a ciaD mutant (Panel I-L), and a ciaD complemented isolate (Panel M-P). Images from left to right show, DAPI staining of cell nuclei (Panels A, E, I, and M), EGFP-cortactin (Panel B, F, J, and N), C. jejuni staining with a polyclonal rabbit a-Campylobacter antibody and a secondary Texas-Red (Panels C, G, K, and O), and merge of all panels (Panels $D, H, L$, and $P$ ). . C. jejuni in contact with the host cell is shown in Panels $\mathrm{H}-1, \mathrm{~L}-1$, and $\mathrm{P}-1$. Images were taken with a $63 \times$ objective and have a $10 \mu \mathrm{M}$ scale bar (Panels A-P). Arrows indicate C. jejuni interaction with host cells. The areas within the boxes highlight regions of membrane ruffling (Panels $\mathrm{F}$ and $\mathrm{N}$ ) and the areas within the circles indicate regions of no membrane ruffling (Panels $B$ and J).

\section{Abbreviations}

ERK: Extracellular regulated kinase; MAPK: Mitogen activated protein kinase; N-WASP: Neuronal Wiskott-Aldrich protein; Cia: Campylobacter invasion antigens.

\section{Competing interests}

Authors declare no conflicts of interest.

\section{Authors' contributions}

DRS planned experiments, performed experiments, and wrote the manuscript. MEK planned experiments, analyzed data, and contributed to preparation of the manuscript. Both authors read and approved the final manuscript.

\section{Acknowledgements}

We would like to thank Jason L. O'Loughlin for performing the immunoprecipitation experiments and Tyson Eucker for performing the Rac1 and $\mathrm{Cdc42}$ activation assays. We also thank the members of the Konkel lab: Jason Neal-McKinney, Jennifer Stone, and Chris Gourley for review of the manuscript. Finally, we thank the Franceschi Microscopy \& Imaging Center at Washington State University for use of the electron and confocal microscope

Funding for this work was provided to MEK by the National Institutes of Health (NIH) grant \#R56 AlO88518-01A1. DRS was partially funded by the National Institutes of General Medical Sciences (NIGMS) Training Grant \#T32GM083864. The content of this manuscript does not represent the official views of the $\mathrm{NIH}$ or NIGMS.

Received: 23 July 2013 Accepted: 23 October 2013

Published: 4 November 2013

\section{References}

1. Selbach M, Backert S: Cortactin: an Achilles' heel of the actin cytoskeleton targeted by pathogens. Trends Microbio/ 2005, 13:181-189.

2. Agerer F, Lux S, Michel A, Rohde M, Ohlsen K, Hauck CR: Cellular invasion by Staphylococcus aureus reveals a functional link between focal adhesion kinase and cortactin in integrin-mediated internalisation. J Cell Sci 2005, 118:2189-2200.

3. Bougneres L, Girardin SE, Weed SA, Karginov AV, Olivo-Marin JC, Parsons JT, Sansonetti PJ, Van Nhieu GT: Cortactin and Crk cooperate to trigger actin polymerization during Shigella invasion of epithelial cells. J Cell Biol 2004, 166:225-235.

4. Rosales EM, Aguilera MO, Salinas RP, Carminati SA, Colombo MI, Martinez-Quiles N, Beron W: Cortactin is involved in the entry of Coxiella burnetii into non-phagocytic cells. PLoS One 2012, 7:e39348.

5. Slanina H, Hebling $S$, Hauck CR, Schubert-Unkmeir A: Cell invasion by Neisseria meningitidis requires a functional interplay between the focal adhesion kinase, Src and cortactin. PLoS One 2012, 7:e39613.

6. Sousa S, Cabanes D, Bougneres L, Lecuit M, Sansonetti $P$, Tran-Van-Nhieu G, Cossart P: Src, cortactin and Arp2/3 complex are required for E-cadherinmediated internalization of Listeria into cells. Cell Microbiol 2007, 9:2629-2643.

7. Cantarelli W, Kodama T, Nijstad N, Abolghait SK, lida T, Honda T: Cortactin is essential for F-actin assembly in enteropathogenic Escherichia coli (EPEC)- and enterohaemorrhagic E. coli (EHEC)induced pedestals and the alpha-helical region is involved in the localization of cortactin to bacterial attachment sites. Cell Microbiol 2006, 8:769-780.

8. Nieto-Pelegrin E, Martinez-Quiles N: Distinct phosphorylation requirements regulate cortactin activation by TirEPEC and its binding to N-WASP. Cell Commun Signal 2009, 7:11.

9. Samuel MC, Vugia DJ, Shallow S, Marcus R, Segler S, McGivern T, Kassenborg H, Reilly K, Kennedy M, Angulo F, Tauxe RV: Epidemiology of sporadic Campylobacter infection in the United States and declining trend in incidence, foodnet 1996-1999. Clin Infect Dis 2004, 38(Suppl 3):S165-S174.

10. Schwerer B: Antibodies against gangliosides: a link between preceding infection and immunopathogenesis of Guillain-Barre syndrome. Microbes Infect 2002, 4:373-384.

11. Christensen JE, Pacheco SA, Konkel ME: Identification of a Campylobacter jejuni-secreted protein required for maximal invasion of host cells. Mol Microbiol 2009, 73:650-662.

12. Konkel ME, Joens LA: Adhesion to and invasion of HEp-2 cells by Campylobacter spp. Infect Immun 1989, 57:2984-2990.

13. Konkel ME, Corwin MD, Joens LA, Cieplak W: Factors that influence the interaction of Campylobacter jejuni with cultured mammalian cells. J Med Microbiol 1992, 37:30-37. 
14. Konkel ME, Kim BJ, Rivera-Amill V, Garvis SG: Bacterial secreted proteins are required for the internaliztion of Campylobacter jejuni into cultured mammalian cells. Mol Microbiol 1999, 32:691-701.

15. Konkel ME, Kim BJ, Rivera-Amill V, Garvis SG: Identification of proteins required for the internalization of Campylobacter jejuni into cultured mammalian cells. Adv Exp Med Biol 1999, 473:215-224.

16. Konkel ME, Klena JD, Rivera-Amill V, Monteville MR, Biswas D, Raphael B, Mickelson J: Secretion of virulence proteins from Campylobacter jejuni is dependent on a functional flagellar export apparatus. J Bacterio/ 2004, 186:3296-3303.

17. Konkel ME, Cieplak W Jr: Altered synthetic response of Campylobacter jejuni to cocultivation with human epithelial cells is associated with enhanced internalization. Infect Immun 1992, 60:4945-4949.

18. Konkel ME, Mead DJ, Cieplak W Jr: Kinetic and antigenic characterization of altered protein synthesis by Campylobacter jejuni during cultivation with human epithelial cells. J Infect Dis 1993, 168:948-954.

19. Neal-McKinney JM, Konkel ME: The Campylobacter jejuni CiaC virulence protein is secreted from the flagellum and delivered to the cytosol of host cells. Front Cell Infect Microbiol 2012, 2:31.

20. Buelow DR, Christensen JE, Neal-McKinney JM, Konkel ME: Campylobacter jejuni survival within human epithelial cells is enhanced by the secreted protein Cial. Mol Microbiol 2011, 80:1296-1312.

21. Konkel ME, Monteville MR, Rivera-Amill V, Joens LA: The pathogenesis of Campylobacter jejuni-mediated enteritis. Curr Issues Intest Microbiol 2001, 2:55-71.

22. Samuelson RD, Tyson PE, Julia AB, Leslie D, Linda SM, Konkel EM: The Campylobacter jejuni CiaD effector protein activates MAP kinase signaling pathways to promote disease. Cell Commun and Signal 2013, 11:79. Submitted.

23. Lee BC, Kim MS, Choi SH, Kim TS: Involvement of capsular polysaccharide via a TLR2/NF-kappaB pathway in Vibrio vulnificus-induced IL-8 secretion of human intestinal epithelial cells. Int J Mol Med 2010, 25:581-591.

24. Hu L, McDaniel JP, Kopecko DJ: Signal transduction events involved in human epithelial cell invasion by Campylobacter jejuni 81-176. Microb Pathog 2006, 40:91-100.

25. MacCallum A, Haddock G, Everest PH: Campylobacter jejuni activates mitogen-activated protein kinases in Caco-2 cell monolayers and in vitro infected primary human colonic tissue. Microbiology 2005, 151:2765-2772.

26. Small JV, Stradal T, Vignal E, Rottner K: The lamellipodium: where motility begins. Trends Cell Biol 2002, 12:112-120.

27. Eucker TP, Konkel ME: The cooperative action of bacterial fibronectinbinding proteins and secreted proteins promote maximal Campylobacter jejuni invasion of host cells by stimulating membrane ruffling. Cell Microbiol 2012, 14:226-238.

28. Krause-Gruszczynska M, Rohde M, Hartig R, Genth $H$, Schmidt G, Keo T, Konig W, Miller WG, Konkel ME, Backert S: Role of the small Rho GTPases Rac1 and Cdc42 in host cell invasion of Campylobacter jejuni. Cell Microbiol 2007, 9:2431-2444

29. Yankulov K, Yamashita K, Roy R, Egly J-M, Bentley DL: The transcriptional elongation inhibitor 5,6-dichloro-1- $\beta$-D-ribofuranosylbenzimidazole inhibits transcription factor $\mathrm{IH}$-associated protein kinase. J Biol Chem 1995, 13;270:23922-23925.

30. Fierer J, Eckmann L, Kagnoff M: IL-8 secreted by epithelial cells invaded by bacteria. Infect Agents Dis 1993, 2:255-258.

31. Hickey TE, Baqar S, Bourgeois AL, Ewing CP, Guerry P: Campylobacter jejunistimulated secretion of interleukin-8 by INT407 cells. Infect Immun 1999, 67:88-93.

32. Kim JM, Jung HY, Lee JY, Youn J, Lee CH, Kim KH: Mitogen-activated protein kinase and activator protein- 1 dependent signals are essential for Bacteroides fragilis enterotoxin-induced enteritis. Eur J Immunol 2005 35:2648-2657

33. Matlawska-Wasowska K, Finn R, Mustel A, O'Byrne CP, Baird AW, Coffey ET, Boyd A: The Vibrio parahaemolyticus type III secretion systems manipulate host cell MAPK for critical steps in pathogenesis. BMC Microbiol 2010, 10:329.

34. Okuda J, Toyotome T, Kataoka N, Ohno M, Abe H, Shimura Y, Seyedarabi A Pickersgill R, Sasakawa C: Shigella effector IpaH9.8 binds to a splicing factor U2AF(35) to modulate host immune responses. Biochem Biophys Res Commun 2005, 333:531-539.

35. Zurawski DV, Mumy KL, Faherty CS, McCormick BA, Maurelli AT: Shigella flexneri type III secretion system effectors OspB and OspF target the nucleus to downregulate the host inflammatory response via interactions with retinoblastoma protein. Mol Microbio/ 2009, 71:350-368.

36. Cosen-Binker LI, Kapus A: Cortactin: the gray eminence of the cytoskeleton. Physiology (Bethesda) 2006, 21:352-361.

37. Campbell DH, Sutherland RL, Daly RJ: Signaling pathways and structural domains required for phosphorylation of EMS1/cortactin. Cancer Res 1999, 15;59:5376-5385

38. Kelley LC, Hayes KE, Ammer AG, Martin KH, Weed SA: Cortactin phosphorylated by Erk 1/2 localizes to sites of dynamic actin regulation and is required for carcinoma lamellipodia persistence. PLoS One 2010, 4;5:e13847.

39. Kelley LC, Hayes KE, Ammer AG, Martin KH, Weed SA: Revisiting the ERK/Src cortactin switch. Commun Integr Biol 2011, 4:205-207.

40. Martinez-Quiles N, Ho HY, Kirschner MW, Ramesh N, Geha RS: Erk/Src phosphorylation of cortactin acts as a switch on-switch off mechanism that controls its ability to activate N-WASP. Mol Cell Biol 2004, 24:5269-5280.

41. Mendoza MC, Er EE, Blenis J: ERK-MAP Kinase signaling in the cytoplasm. Methods Mol Biol 2010, 661:185-203.

42. Roskoski R Jr: Erk 1/2 MAP kinases: structure, function, and regulation. Pharmacol Res 2012, 66:105-143.

43. Konkel ME, Garvis SG, Tipton SL, Anderson DE Jr, Cieplak W Jr: Identification and molecular cloning of a gene encoding a fibronectin-binding protein (CadF) from Campylobacter jejuni. Mol Microbiol 1997, 24:953-963.

44. Flanagan RC, Neal-McKinney JM, Dhillon AS, Miller WG, Konkel ME: Examination of Campylobacter jejuni putative adhesins leads to the identification of a new protein, designated FlpA, required for chicken colonization. Infect Immun 2009, 77:2399-2407.

45. Krause-Gruszczynska M, Boehm M, Rohde M, Tegtmeyer N, Takahashi S, Buday L, Oyarzabal OA, Backert S: The signaling pathway of Campylobacter jejuni-induced Cdc42 activation: Role of fibronectin, integrin beta1, tyrosine kinases and guanine exchange factor Vav2. In Cell commun signal: volume 9. England; 2011:32.

46. Monteville MR, Yoon JE, Konkel ME: Maximal adherence and invasion of INT 407 cells by Campylobacter jejuni requires the CadF outer-membrane protein and microfilament reorganization. Microbiology 2003, 149:153-165.

47. Rivera-Amill V, Kim BJ, Seshu J, Konkel ME: Secretion of the virulenceassociated Campylobacter invasion antigens from Campylobacter jejuni requires a stimulatory signal. J Infect Dis 2001, 183:1607-1616.

48. Zhou D, Galan J: Salmonella entry into host cells: the work in concert of type III secreted effector proteins. Microbes Infect 2001, 3:1293-1298.

49. Rodriguez-Escudero I, Ferrer NL, Rotger R, Cid VJ, Molina M: Interaction of the Salmonella Typhimurium effector protein SopB with host cell Cdc42 is involved in intracellular replication. Mol Microbio/ 2011, 80:1220-1240.

50. Egile C, Loisel TP, Laurent V, Li R, Pantaloni D, Sansonetti PJ, Carlier MF: Activation of the CDC42 effector N-WASP by the Shigella flexneri IcsA protein promotes actin nucleation by Arp2/3 complex and bacterial actin-based motility. J Cell Biol 1999, 146:1319-1332.

51. Romero S, Grompone G, Carayol N, Mounier J, Guadagnini S, Prevost MC, Sansonetti PJ, Van Nhieu GT: ATP-mediated Erk1/2 activation stimulates bacterial capture by filopodia, which precedes Shigella invasion of epithelial cells. Cell Host Microbe 2011, 9:508-519.

52. Li H, Xu H, Zhou Y, Zhang J, Long C, Li S, Chen S, Zhou JM, Shao F: The phosphothreonine lyase activity of a bacterial type III effector family. Science 2007, 315:1000-1003.

53. Mounier J, Popoff MR, Enninga J, Frame MC, Sansonetti PJ, Van Nhieu GT: The IpaC carboxyterminal effector domain mediates Src-dependent actin polymerization during Shigella invasion of epithelial cells. PLOS Pathog 2009, 5:e1000271.

doi:10.1186/1478-811X-11-82

Cite this article as: Samuelson and Konkel: Serine phosphorylation of cortactin is required for maximal host cell invasion by Campylobacter jejuni. Cell Communication and Signaling 2013 11:82. 